\title{
Fatores associados às práticas assistenciais ao recém-nascido na sala de parto
}

\author{
Factors associated with care practices for newborns in the delivery room \\ Factores asociados a las prácticas de asistencia al recién nacido en la sala de parto
}

\begin{abstract}
Beatriz Cabral Ledo ${ }^{1}$ (c)
Fernanda Garcia Bezerra Góes ${ }^{1}$ (1)

Andressa Silva Torres dos Santos ${ }^{1}$ (B) Fernanda Maria Vieira Pereira-Ávila ${ }^{1}$ (B) Aline Cerqueira Santos Santana da Silva ${ }^{1}$ (]) Mayara Pacheco da Conceição Bastos ${ }^{1}$ (10)

1.Universidade Federal Fluminense, Instituto de Humanidades e Saúde. Rio das Ostras, RJ, Brasil.
\end{abstract}

Autor Correspondente:

Fernanda Garcia Bezerra Góes

E-mail: ferbezerra@gmail.com

Recebido em 26/03/2020

Aprovado em 17/06/2020

\section{RESUMO}

Objetivo: identificar os fatores associados às práticas assistenciais ao recém-nascido adotadas na sala de parto de uma maternidade na baixada litorânea do Rio de Janeiro. Método: estudo transversal, realizado em instituição pública no estado Rio de Janeiro, mediante coleta de dados em prontuários de nascimentos entre 2015 e 2017. Na associação entre variáveis, adotou-se o Teste Qui-Quadrado e a regressão logística. Resultados: entre 351 (100,0\%) prontuários, constituíram-se como práticas realizadas na sala de parto: contato pele a pele e aleitamento materno precoce (28,0\%); secagem (92,3\%); aspiração oronasofaríngea (82,1\%); aspiração gástrica (52,7\%); aspiração traqueal (12,2\%); oxigênio inalatório (7,7\%); e encaminhamento ao Alojamento Conjunto $(91,1 \%)$. O contato precoce com o seio materno esteve associado ao tipo de parto $(p=0,043)$ e às alterações no exame físico $(p=0,001)$. Possuir alterações no exame físico ao nascimento diminuiu significativamente as chances de o bebê ser colocado nessa posição ainda na sala de parto $(\mathrm{p}=0,001)$ assim como os recém-nascidos de parto cesáreo $(\mathrm{p}=0,045)$. Nascer de cesárea aumentou duas vezes as chances de o recém-nascido ser submetido à aspiração gástrica $(p=0,002)$. Conclusão e implicações para a prática: é premente organizar as rotinas dos serviços, de modo a evitar intervenções desnecessárias visando uma atenção obstétrica e neonatal humanizada e de qualidade.

Palavras-chave: Recém-Nascido; Cuidado; Aleitamento Materno; Parto Humanizado; Enfermagem Neonatal

\section{ABSTRACT}

Objective: to identify the factors associated with newborn care practices adopted in the delivery room of a maternity hospital in the coastal lowlands of Rio de Janeiro. Method: a cross-sectional was study carried out in a public institution in the state of Rio de Janeiro using data collected from birth records between 2015 and 2017. The chi-square test and logistic regression were adopted to associate the variables. Results: among 351 (100.0\%) medical records, the following constituted practices performed in the delivery room: skin-to-skin contact and early breastfeeding (28.0\%); drying (92.3\%); oronasopharyngeal aspiration (82.1\%); gastric aspiration (52.7\%); tracheal aspiration (12.2\%); inhaled oxygen (7.7\%); and rooming-in referral (91.1\%). Early breastfeeding was associated with the type of delivery $(p=0.043)$ and changes in physical examination $(p=0.001)$. Changes in the physical examination at birth significantly decreased the chances of babies being placed in this position while still in the delivery room ( $p=0.001)$, as well as newborns delivered by cesarean section $(p=0.045)$. Being born by cesarean section increased the chances of newborns being submitted to gastric aspiration twice $(p=0.002)$. Conclusion and implications for practice: it is urgent to organize the routines of services in order to avoid unnecessary interventions aiming at humanized and quality obstetric and neonatal care.

Keywords: Infant; Newborn; Care; Breastfeeding; Humanizing Delivery; Neonatal Nursing

\section{RESUMEN}

Objetivo: identificar los factores asociados con las prácticas de atención para recién nacidos adoptados en la sala de partos de un hospital de maternidad en las tierras bajas costeras de Rio de Janeiro. Método: estudio transversal, realizado en una institución pública en el estado de Rio de Janeiro, utilizando datos de registros de nacimiento entre 2015 y 2017 . Se adoptó la prueba de chi-cuadrado y la regresión logística en la asociación entre variables. Resultados: entre 351 (100.0\%) registros médicos, se realizaron las siguientes prácticas en la sala de partos: contacto piel con piel y lactancia temprana (28.0\%); secado $(92,3 \%)$; aspiración oronasofaríngea (82,1\%); aspiración gástrica $(52.7 \%)$; aspiración traqueal (12,2\%); oxígeno inhalado (7.7\%); y derivación a Vivienda Conjunta (91.1\%). El contacto con el seno materno temprano se asoció con el tipo de parto $(p=0.043)$ y los cambios en el examen físico $(p=0.001)$. Tener cambios en el examen físico al nacer redujo significativamente las posibilidades de colocar al bebé en esta posición mientras aún está en la sala de partos ( $p=0.001)$, así como a los recién nacidos por cesárea $(\mathrm{p}=0.045)$. Nacer por cesárea aumentó las posibilidades de que el recién nacido sea sometido a aspiración gástrica dos veces $(\mathrm{p}=0.002)$. Conclusión e implicaciones para la práctica: es urgente organizar las rutinas de los servicios para evitar intervenciones innecesarias a través de una atención obstétrica y neonatal humanizada y de calidad.

Palabras clave: Recién Nacido; Cuidado; Lactancia Materna; Parto Humanizado; Enfermería Neonatal. 


\section{INTRODUÇÃO}

A transição no momento do parto para a vida extrauterina envolve uma série de alterações fisiológicas no recém-nascido, inclusive no padrão de trocas gasosas, no equilíbrio ácido básico e na atividade cardiovascular. ${ }^{1}$ Em vista disso, o atendimento ao recém-nascido na sala de parto requer a adoção de cuidados que favoreçam a adaptação à vida extrauterina com o mínimo de interferências possíveis, ${ }^{2}$ a partir da fisiologia do trabalho de parto, com a utilização de tecnologias de cuidado humanizado que proporcionem conforto e segurança para o desenvolvimento da saúde imediata e futura da criança. ${ }^{3}$

As diretrizes de humanização do parto quanto à atuação profissional caracterizam-se por um conjunto de condutas que visam à promoção do parto humanizado e nascimento saudáveis, respeitando a mulher e o recém-nascido, com práticas não intervencionistas. ${ }^{4}$ Assim, no que tange à primeira hora de vida do recém-nascido logo após o nascimento, considerada a hora dourada, é preciso estimular a formação do vínculo entre mãe e bebê. Nesse primeiro momento, vislumbrando o cuidado humanístico, evidências apontam para a importância da promoção do contato pele a pele, da amamentação na primeira hora, do clampeamento oportuno do cordão umbilical, da participação do acompanhante nas salas de parto, ${ }^{3,5}$ dentre outras práticas.

O contato precoce pele a pele da mãe com o bebê auxilia a formação do vínculo entre a díade; ${ }^{6-8}$ na amamentação na primeira hora de vida; na estabilização do sistema cardiorrespiratório; na saturação de oxigênio do bebê;e na regulação da temperatura e do choro. ${ }^{6}$ A amamentação precoce é essencial dados os benefícios imunológicos e psicossociais, ${ }^{9,10}$ além de prevenir a morbimortalidade neonatal ${ }^{10} \mathrm{e}$ o desmame precoce. ${ }^{6} \mathrm{O}$ clampeamento oportuno do cordão umbilical reduz o risco de anemia na primeira infância, ${ }^{3}$ e o acompanhante é reconhecido enquanto promotor da aplicação das boas práticas durante o parto. ${ }^{11}$

Contudo, poucos estudos produzidos possuem o foco nas práticas assistenciais ao recém-nascido nas salas de parto, pois, geralmente, estão atrelados à saúde da mulher, possuindo uma abordagem superficial relativa aos cuidados com o bebê. ${ }^{3,12,13}$ Além disso, não elencam fatores locais associados à ocorrência ou não das boas práticas recomendadas pela Organização Mundial de Saúde (OMS) por parte dos profissionais que prestam tal assistência. $^{2}$

Mundialmente, existem variações e divergências quanto ao atendimento ao parto e ao recém-nascido na primeira hora de vida devido às desigualdades geográficas e sociais e às diferenças nas condutas profissionais e nas características demográficas da clientela. Esses fatores podem afetar diretamente a qualidade do cuidado ofertado e alterar os resultados neonatais imediatos e ao longo da vida. No Brasil, o modelo predominante de atenção ao parto e nascimento ainda é tecnocrático, centrado no profissional, repleto de intervenções inadequadas e desnecessárias, tornando o nascimento um evento patológico. ${ }^{2}$

Desta forma, justifica-se a realização de estudos locais que visem analisar as práticas assistenciais junto aos recém-nascidos em distintas salas de parto, frente às melhores evidências científicas disponíveis na atualidade. Assim, o presente estudo tem como objetivo identificar fatores associados às práticas assistenciais, ao recém-nascido, adotadas na sala de parto de uma maternidade na baixada litorânea do Rio de Janeiro.

\section{MÉTODO}

Estudo transversal, realizado em uma instituição pública de médio porte localizada na região da baixada litorânea do estado do Rio de Janeiro, Brasil. Vale ressaltar que é a única maternidade pública do município que presta assistência à população do território de abrangência (aproximadamente 106.000 habitantes), além das cidades do entorno. A unidade possui 20 leitos destinados ao Alojamento Conjunto e quatro leitos na unidade intermediária neonatal; ademais, não possui banco de leite, unidade canguru e terapia intensiva neonatal.

A coleta de dados foi realizada entre abril de 2018 e julho de 2019, mediante consulta aos prontuários de nascimentos ocorridos no período de 2015 e 2017, utilizando-se de um formulário estruturado, previamente elaborado pelas autoras, com todas as variáveis do tipo categóricas, com exceção do peso, que era contínua.

As variáveis dependentes foram relacionadas às práticas assistenciais realizadas com o recém-nascido na sala de parto e incluíram contato com o seio materno, secagem e encaminhamento ao Alojamento Conjunto, além das intervenções invasivas, como aspirações oronasofaríngea, gástrica e traqueal e oxigênio inalatório. O clampeamento oportuno do cordão umbilical e a participação do acompanhante não foram incluídos pela ausência desses dados registrados nos documentos. As variáveis independentes avaliadas foram: faixa etária e escolaridade materna, realização de pré-natal e número de consultas, tipo de gestação e de parto, presença de gravidez de risco e de intercorrências na gestação; e as variáveis relacionadas ao recém-nascido foram: sexo, raça/cor, idade gestacional, casos de sofrimento fetal, realização de manobras de reanimação, Apgar 1'/5', peso/idade gestacional e alterações no exame físico.

De acordo com informações obtidas junto à instituição, no período compreendido entre 2015 e 2017, foram totalizados 4.000 nascimentos. Para a seleção dos prontuários, foi realizado um cálculo amostral adotando-se o intervalo de confiança de $95 \%$, erro amostral de $5 \%$ e estimativa de prevalência de 50\%, totalizando uma amostra de 351 nascimentos.

Foram incluídos, aleatoriamente, os prontuários com dados de recém-nascidos cujos partos ocorreram no cenário de pesquisa no período de estudo. Prontuários não localizados ou indisponíveis para acesso foram excluídos. A consulta ocorreu em um ambiente reservado da própria instituição, respeitando-se a rotina e o funcionamento do serviço.

Os dados coletados foram digitados em planilha do programa Microsoft Office Excel ${ }^{\circledR}$, mediante dupla digitação e validação. Esses foram importados para o software IBM ${ }^{\circledR}$ SPSS, versão 21.0, e submetidos à análise estatística descritiva, com medidas de frequência absoluta para todas as variáveis categóricas, e tendência central (média, mediana) e de dispersão (desvio-padrão) 
para o peso. Para alterações no exame físico, considerou-se o recém-nascido ter apresentado, ao menos, uma alteração. Para a análise da associação entre as variáveis dependentes e independentes, adotou-se o Teste Qui-Quadrado. Em seguida, as associações estatisticamente significantes foram submetidas à regressão logística binária simples, na medida em que as variáveis dependentes eram de natureza dicotômica, visando, assim, estimar as probabilidades coligadas à ocorrência de cada uma das práticas assistenciais, por meio do cálculo de Odds Ratio (OR) e seus respectivos intervalos de confiança de $95 \%$. O nível de significância foi de $5 \%$.

O projeto ao qual este estudo está vinculado foi aprovado pelo Comitê de Ética em Pesquisa (CAAE 82844818.5.0000.5243; Parecer 2.564.375), a saber: "Perfil clínico e sociodemográfico de recém-nascidos de uma maternidade pública em Rio das Ostras". A obtenção dos dados dos prontuários ocorreu mediante a assinatura do Termo de Compromisso para Utilização de Dados (TCDU) pelas pesquisadoras.

\section{RESULTADOS}

Um total de 351 (100,0\%) prontuários de nascimentos foi analisado. A maior parcela foi de mães entre 20 e 34 anos, (227; $67,7 \%)$, seguido de mães de até 19 anos, $(75 ; 22,4 \%)$, e de 35 anos ou mais (33; 9,9\%). A escolaridade das mães com ensino médio (195; 58,7\%) apresentou maior proporção, seguido do ensino fundamental $(113 ; 34,0 \%)$, ensino superior $(22 ; 6,6 \%)$ e analfabetas $(02 ; 0,6 \%)$. A maioria era residente do município de Rio das Ostras (293; 84,2\%).

Quanto às condições gestacionais, 329 (97,9\%) foram gestações únicas e a maioria das mães realizou pré-natal com acompanhamento de baixo risco $(211 ; 80,8 \%)$, sem intercorrências $(155 ; 57,8 \%)$. O parto cesáreo foi prevalente ( $188 ; 54,0 \%)$, com pequeno predomínio de bebês do sexo feminino (177; 50,9\%). Em relação à raça/cor, (142; 52,0\%) eram brancos. Quanto à idade gestacional, (302; 86\%) tinham entre 37 e 41 semanas; ademais, $324(92,8 \%)$ e $348(99,4 \%)$, respectivamente, apresentaram Apgar no $1^{\circ}$ e no $5^{\circ}$ minuto superior a sete. A maioria $(280 ; 87,2 \%)$ foi classificada com peso adequado para idade gestacional (Tabela 1). A média de peso ao nascer foi de $3.219,9 \mathrm{~g}(\mathrm{DP}=566,3)$, com mínimo de $1.115 \mathrm{~g}$ e máximo de $5.180 \mathrm{~g}$.

Com relação às alterações no exame físico, $110(32,7 \%)$ recém-nascidos apresentaram algum tipo ou mais. Dentre essas, as mais frequentes foram: cianose $(40 ; 36,4 \%)$, bossa serosanguínea $(31 ; 28,2 \%)$, desconforto respiratório $(17 ; 15,5 \%)$ e lesões de pele (17; 15,5\%).

No tocante às práticas assistenciais realizadas na sala de parto, em relação às ações recomendadas, a maior parte foi submetida à secagem $(289 ; 92,3 \%)$, porém apenas $67(28,0 \%)$ dos recém-nascidos entraram em contato pele a pele e foram levados ao seio materno logo após o nascimento. Enquanto isso, $296(91,1 \%)$ foram encaminhados ao Alojamento Conjunto junto de suas mães após a saída da sala de parto, por possuírem boa vitalidade.
Tabela 1 - Caracterização dos recém-nascidos segundo variáveis gestacionais, individuais e clínicas ( $\mathrm{N}=351$ ). Rio das Ostras, $\mathrm{RJ}$, Brasil, 2019.

\begin{tabular}{|c|c|}
\hline Variáveis & n (\%) \\
\hline \multicolumn{2}{|l|}{ Sexo* } \\
\hline Feminino & $177(50,9)$ \\
\hline Masculino & $171(49,1)$ \\
\hline \multicolumn{2}{|l|}{ Raça/cor* } \\
\hline Preta & $9(3,3)$ \\
\hline Parda & $122(44,7)$ \\
\hline Branca & $142(52,0)$ \\
\hline \multicolumn{2}{|c|}{ Idade gestacional no nascimento* } \\
\hline Pré-termo & $22(6,3)$ \\
\hline A termo & $302(86,0)$ \\
\hline Pós-termo & $1(0,3)$ \\
\hline \multicolumn{2}{|c|}{ Sofrimento fetal diagnosticado* } \\
\hline Não & $229(94,6)$ \\
\hline Sim & $13(5,4)$ \\
\hline \multicolumn{2}{|l|}{ Apgar $1^{\prime *}$} \\
\hline 0 a 3 & $3(0,9)$ \\
\hline 4 a 6 & $22(6,3)$ \\
\hline 7 a 10 & $324(92,8)$ \\
\hline \multicolumn{2}{|l|}{ Apgar $5^{*}$} \\
\hline 0 a 3 & $1(0,3)$ \\
\hline 4 a 6 & $1(0,3)$ \\
\hline 7 a 10 & $348(99,4)$ \\
\hline \multicolumn{2}{|c|}{ Peso/idade gestacional* } \\
\hline PIG & $12(3,7)$ \\
\hline AIG & $280(87,2)$ \\
\hline GIG & $29(9,0)$ \\
\hline \multicolumn{2}{|l|}{ Tipo de gestação* } \\
\hline Única & $329(97,9)$ \\
\hline Múltipla & $7(2,1)$ \\
\hline \multicolumn{2}{|l|}{ Tipo de parto* } \\
\hline Vaginal & $160(46,0)$ \\
\hline Cesáreo & $188(54,0)$ \\
\hline \multicolumn{2}{|l|}{ Pré-natal* } \\
\hline Nenhuma consulta & $8(2,4)$ \\
\hline Até cinco consultas & $100(31,9)$ \\
\hline Mais de seis & $213(68,0)$ \\
\hline \multicolumn{2}{|c|}{ Intercorrências na gestação* } \\
\hline Não & $155(57,8)$ \\
\hline Sim & $113(42,2)$ \\
\hline \multicolumn{2}{|c|}{ Alterações no exame físico* } \\
\hline Não & $226(67,3)$ \\
\hline Sim & $110(32,7)$ \\
\hline
\end{tabular}

Nota: GIG - grande para a idade gestacional; AIG - adequado para a idade gestacional; PIG - pequeno para a idade gestacional. * Variável apresentou missing. 
O contato precoce com o seio materno esteve associado ao tipo de parto $(p=0,043)$ e às alterações no exame físico $(p=0,001)$. Ainda, houve associação entre o encaminhamento ao Alojamento Conjunto e gravidez de risco $(p=0,012)$, sofrimento fetal $(p=0,013)$, alterações no exame físico $(p=0,000)$ e manobras de reanimação $(p=0,000)$ (Tabela 2$)$.

Dadas as características de baixo risco desses recém-nascidos, considerando as características gestacionais, individuais e clínicas ao nascimento, as frequências de aplicação de intervenções invasivas foram elevadas e incluíram aspiração oronasofaríngea $(257 ; 82,1 \%)$, aspiração gástrica $(165 ; 52,7 \%)$, aspiração traqueal $(38 ; 12,2 \%)$ e oxigênio inalatório $(24 ; 7,7 \%)$. Não houve associação das práticas recomendadas e invasivas com as variáveis demográficas.
Contudo, dentre essas práticas, a aspiração oronasofaríngea apresentou associação estatística entre as variáveis alterações no exame físico $(p=0,049)$ e manobras de reanimação $(p=0,009)$; aspiração gástrica ao tipo de parto $(p=0,002)$; aspiração traqueal às manobras de reanimação $(p=0,031)$; e oxigênio inalatório às alterações no exame físico do recém-nascido $(p=0,000)$ e às manobras de reanimação $(p=0,000)$ (Tabela 3$)$.

Na regressão logística binária, em relação às práticas recomendadas, as variáveis tipo de parto e alterações no exame físico permaneceram associadas ao contato com o seio materno. Observou-se que possuir alterações no exame físico ao nascimento diminuiu significativamente as chances de o bebê ser colocado nessa posição na sala de parto $(p=0,001 ; O R=0,32 ; \mathrm{IC}: 0,167-0,632)$, em relação aos sem

Tabela 2 - Associação entre práticas assistenciais recomendadas e variáveis clínicas e demográficas (N=351). Rio das Ostras, RJ, Brasil, 2019.

\begin{tabular}{|c|c|c|c|c|c|c|c|c|c|}
\hline \multirow{3}{*}{ Variáveis } & \multicolumn{2}{|c|}{$\begin{array}{c}\text { Contato com o seio } \\
\text { materno }\end{array}$} & \multirow{3}{*}{$P^{* *}$} & \multicolumn{2}{|c|}{ Secagem } & \multirow{3}{*}{$P^{* *}$} & \multicolumn{2}{|c|}{$\begin{array}{c}\text { Alojamento } \\
\text { Conjunto }\end{array}$} & \multirow{3}{*}{$P^{* *}$} \\
\hline & Não & Sim & & Não & Sim & & Não & Sim & \\
\hline & $\begin{array}{c}n \\
(\%)\end{array}$ & $\begin{array}{c}n \\
(\%)\end{array}$ & & $\begin{array}{c}n \\
(\%)\end{array}$ & $\begin{array}{c}n \\
(\%)\end{array}$ & & $\begin{array}{c}n \\
(\%)\end{array}$ & $\begin{array}{c}n \\
(\%)\end{array}$ & \\
\hline Sexo* & & & 0,157 & & & 0,436 & & & 0,371 \\
\hline Feminino & $\begin{array}{c}77 \\
(45)\end{array}$ & $\begin{array}{c}37 \\
(55,2)\end{array}$ & & $\begin{array}{c}10 \\
(43,5)\end{array}$ & $\begin{array}{c}149 \\
(51,9)\end{array}$ & & $\begin{array}{c}12 \\
(7,3)\end{array}$ & $\begin{array}{c}152 \\
(92,7)\end{array}$ & \\
\hline Masculino & $\begin{array}{c}94 \\
(55)\end{array}$ & $\begin{array}{c}30 \\
(44,8)\end{array}$ & & $\begin{array}{c}13 \\
(56,5)\end{array}$ & $\begin{array}{c}138 \\
(48,1)\end{array}$ & & $\begin{array}{c}16 \\
(10,1)\end{array}$ & $\begin{array}{c}142 \\
(89,9)\end{array}$ & \\
\hline Pré-natal* & & & 0,68 & & & 0,65 & & & 0,362 \\
\hline Não & $\begin{array}{c}8 \\
(4,9)\end{array}$ & $\begin{array}{c}0 \\
(0)\end{array}$ & & $\begin{array}{c}2 \\
(8,7)\end{array}$ & $\begin{array}{c}6 \\
(2,2)\end{array}$ & & $\begin{array}{c}0 \\
(0)\end{array}$ & $\begin{array}{c}8 \\
(100)\end{array}$ & \\
\hline Sim & $\begin{array}{c}154 \\
(95,1)\end{array}$ & $\begin{array}{c}65 \\
(100)\end{array}$ & & $\begin{array}{c}21 \\
(91,3)\end{array}$ & $\begin{array}{c}267 \\
(97,8)\end{array}$ & & $\begin{array}{c}28 \\
(9,4)\end{array}$ & $\begin{array}{c}269 \\
(90,6)\end{array}$ & \\
\hline Gravidez de risco* & & & 0,107 & & & 0,117 & & & 0,012 \\
\hline Não & $\begin{array}{c}102 \\
(73,4)\end{array}$ & $\begin{array}{c}37 \\
(26,6)\end{array}$ & & $\begin{array}{c}12 \\
(6,4)\end{array}$ & $\begin{array}{c}176 \\
(93,6)\end{array}$ & & $\begin{array}{c}12 \\
(6,1)\end{array}$ & $\begin{array}{c}185 \\
(93,9)\end{array}$ & \\
\hline Sim & $\begin{array}{c}27 \\
(87,1)\end{array}$ & $\begin{array}{c}4 \\
(12,9)\end{array}$ & & $\begin{array}{c}6 \\
(13,3)\end{array}$ & $\begin{array}{c}39 \\
(86,7)\end{array}$ & & $\begin{array}{c}8 \\
(17,4)\end{array}$ & $\begin{array}{c}38 \\
(82,6)\end{array}$ & \\
\hline Intercorrências na gestação* & & & 0,700 & & & 0,471 & & & 0,093 \\
\hline Não & $\begin{array}{c}73 \\
(53,3)\end{array}$ & $\begin{array}{c}23 \\
(50)\end{array}$ & & $\begin{array}{c}8 \\
(50)\end{array}$ & $\begin{array}{c}132 \\
(59,2)\end{array}$ & & $\begin{array}{c}12 \\
(8,1)\end{array}$ & $\begin{array}{c}136 \\
(91,9)\end{array}$ & \\
\hline Sim & $\begin{array}{c}64 \\
(46,7)\end{array}$ & $\begin{array}{c}23 \\
(50)\end{array}$ & & $\begin{array}{c}8 \\
(50)\end{array}$ & $\begin{array}{c}91 \\
(40,8)\end{array}$ & & $\begin{array}{c}15 \\
(14,9)\end{array}$ & $\begin{array}{c}86 \\
(85,1)\end{array}$ & \\
\hline Tipo de parto* & & & 0,043 & & & 0,892 & & & 0,073 \\
\hline Vaginal & $\begin{array}{c}68 \\
(40)\end{array}$ & $\begin{array}{c}36 \\
(54,5)\end{array}$ & & $\begin{array}{c}11 \\
(45,8)\end{array}$ & $\begin{array}{c}127 \\
(44,4)\end{array}$ & & $\begin{array}{c}9 \\
(6)\end{array}$ & $\begin{array}{l}142 \\
(94)\end{array}$ & \\
\hline Cesárea & $\begin{array}{l}102 \\
(60)\end{array}$ & $\begin{array}{c}30 \\
(45,5)\end{array}$ & & $\begin{array}{c}13 \\
(54,2)\end{array}$ & $\begin{array}{c}159 \\
(55,6)\end{array}$ & & $\begin{array}{c}20 \\
(11,7)\end{array}$ & $\begin{array}{c}151 \\
(88,3)\end{array}$ & \\
\hline
\end{tabular}

* Variável apresentou missing. ** Teste estatístico Qui-Quadrado. 
Tabela 2 - Continuação...

\begin{tabular}{|c|c|c|c|c|c|c|c|c|c|}
\hline \multirow{3}{*}{ Variáveis } & \multicolumn{2}{|c|}{$\begin{array}{c}\text { Contato com o seio } \\
\text { materno }\end{array}$} & \multirow{3}{*}{$P * *$} & \multicolumn{2}{|c|}{ Secagem } & \multirow{3}{*}{$p^{* *}$} & \multicolumn{2}{|c|}{$\begin{array}{l}\text { Alojamento } \\
\text { Conjunto }\end{array}$} & \multirow{3}{*}{$P^{* *}$} \\
\hline & Não & Sim & & Não & Sim & & Não & Sim & \\
\hline & $\begin{array}{c}n \\
(\%)\end{array}$ & $\begin{array}{c}n \\
(\%)\end{array}$ & & $\begin{array}{c}n \\
(\%)\end{array}$ & $\begin{array}{c}n \\
(\%)\end{array}$ & & $\begin{array}{c}n \\
(\%)\end{array}$ & $\begin{array}{c}n \\
(\%)\end{array}$ & \\
\hline Sofrimento fetal* & & & 0,542 & & & 0,964 & & & 0,013 \\
\hline Não & $\begin{array}{c}113 \\
(94,2)\end{array}$ & $\begin{array}{c}31 \\
(96,9)\end{array}$ & & $\begin{array}{c}15 \\
(93,8)\end{array}$ & $\begin{array}{l}189 \\
(94)\end{array}$ & & $\begin{array}{l}13 \\
(6)\end{array}$ & $\begin{array}{l}202 \\
(94)\end{array}$ & \\
\hline Sim & $\begin{array}{c}7 \\
(5,8)\end{array}$ & $\begin{array}{c}1 \\
(3,1)\end{array}$ & & $\begin{array}{c}1 \\
(6,3)\end{array}$ & $\begin{array}{l}12 \\
(6)\end{array}$ & & $\begin{array}{c}3 \\
(25)\end{array}$ & $\begin{array}{c}9 \\
(75)\end{array}$ & \\
\hline Alteração no exame físico* & & & 0,001 & & & 0,322 & & & 0,000 \\
\hline Não & $\begin{array}{c}90 \\
(54,2)\end{array}$ & $\begin{array}{c}51 \\
(78,5)\end{array}$ & & $\begin{array}{c}17 \\
(77,3)\end{array}$ & $\begin{array}{l}189 \\
(67)\end{array}$ & & $\begin{array}{c}6 \\
(2,8)\end{array}$ & $\begin{array}{c}208 \\
(97,2)\end{array}$ & \\
\hline Sim & $\begin{array}{c}76 \\
(45,8)\end{array}$ & $\begin{array}{c}14 \\
(21,5)\end{array}$ & & $\begin{array}{c}5 \\
(22,7)\end{array}$ & $\begin{array}{c}93 \\
(33)\end{array}$ & & $\begin{array}{c}23 \\
(22,8)\end{array}$ & $\begin{array}{c}78 \\
(77,2)\end{array}$ & \\
\hline Manobras de reanimação* & & & 0,855 & & & 0,119 & & & 0,000 \\
\hline Não & $\begin{array}{c}139 \\
(87,4)\end{array}$ & $\begin{array}{c}53 \\
(88,3)\end{array}$ & & $\begin{array}{c}19 \\
(100)\end{array}$ & $\begin{array}{c}232 \\
(88,5)\end{array}$ & & $\begin{array}{c}15 \\
(5,8)\end{array}$ & $\begin{array}{c}244 \\
(94,2)\end{array}$ & \\
\hline Sim & $\begin{array}{c}20 \\
(12,6) \\
\end{array}$ & $\begin{array}{c}7 \\
(11,7) \\
\end{array}$ & & $\begin{array}{c}0 \\
(0) \\
\end{array}$ & $\begin{array}{c}30 \\
(11,5) \\
\end{array}$ & & $\begin{array}{c}13 \\
(41,9) \\
\end{array}$ & $\begin{array}{c}18 \\
(58,1) \\
\end{array}$ & \\
\hline
\end{tabular}

* Variável apresentou missing. ** Teste estatístico Qui-Quadrado.

Tabela 3 - Associação entre intervenções invasivas e variáveis clínicas e demográficas (N=351). Rio das Ostras, RJ, Brasil, 2019.

\begin{tabular}{|c|c|c|c|c|c|c|c|c|c|c|c|c|}
\hline \multirow{3}{*}{ Variáveis } & \multicolumn{2}{|c|}{$\begin{array}{c}\text { Aspiração } \\
\text { oronasofaríngea }\end{array}$} & \multirow{3}{*}{$P * *$} & \multicolumn{2}{|c|}{$\begin{array}{l}\text { Aspiração } \\
\text { gástrica }\end{array}$} & \multirow{3}{*}{$P^{* *}$} & \multicolumn{2}{|c|}{$\begin{array}{c}\text { Aspiração } \\
\text { traqueal }\end{array}$} & \multirow{3}{*}{$\boldsymbol{P}^{* *}$} & \multicolumn{2}{|c|}{$\mathrm{O}_{2}$ inalatório } & \multirow{3}{*}{$P^{* *}$} \\
\hline & Não & Sim & & Não & Sim & & Não & Sim & & Não & Sim & \\
\hline & $\begin{array}{c}n \\
(\%)\end{array}$ & $\begin{array}{c}n \\
(\%)\end{array}$ & & $\begin{array}{c}n \\
(\%)\end{array}$ & $\begin{array}{c}n \\
(\%)\end{array}$ & & $\begin{array}{c}n \\
(\%)\end{array}$ & $\begin{array}{c}n \\
(\%)\end{array}$ & & $\begin{array}{c}n \\
(\%)\end{array}$ & $\begin{array}{c}n \\
(\%)\end{array}$ & \\
\hline Sexo * & & & 0,814 & & & 0,377 & & & 0,877 & & & 0,109 \\
\hline Feminino & $\begin{array}{c}29 \\
(18,2)\end{array}$ & $\begin{array}{c}130 \\
(81,8)\end{array}$ & & $\begin{array}{c}71 \\
(44,7)\end{array}$ & $\begin{array}{c}88 \\
(55,3)\end{array}$ & & $\begin{array}{c}139 \\
(87,4)\end{array}$ & $\begin{array}{c}20 \\
(12,6)\end{array}$ & & $\begin{array}{c}143 \\
(94,7)\end{array}$ & $\begin{array}{c}8 \\
(5,3)\end{array}$ & \\
\hline Masculino & $\begin{array}{c}26 \\
(17,2)\end{array}$ & $\begin{array}{c}125 \\
(82,8)\end{array}$ & & $\begin{array}{c}75 \\
(49,7)\end{array}$ & $\begin{array}{c}76 \\
(50,3)\end{array}$ & & $\begin{array}{l}132 \\
(88)\end{array}$ & $\begin{array}{c}18 \\
(12)\end{array}$ & & $\begin{array}{c}141 \\
(89,8)\end{array}$ & $\begin{array}{c}16 \\
(10,2)\end{array}$ & \\
\hline Pré-natal * & & & 0,616 & & & 0,427 & & & 0,244 & & & 0,650 \\
\hline Não & $\begin{array}{c}2 \\
(25)\end{array}$ & $\begin{array}{c}6 \\
(75)\end{array}$ & & $\begin{array}{c}5 \\
(62,5)\end{array}$ & $\begin{array}{c}3 \\
(37,5)\end{array}$ & & $\begin{array}{c}6 \\
(75)\end{array}$ & $\begin{array}{c}2 \\
(25)\end{array}$ & & $\begin{array}{c}7 \\
(87,5)\end{array}$ & $\begin{array}{c}1 \\
(12,5)\end{array}$ & \\
\hline Sim & $\begin{array}{c}52 \\
(18,1)\end{array}$ & $\begin{array}{c}236 \\
(81,9)\end{array}$ & & $\begin{array}{c}139 \\
(48,3)\end{array}$ & $\begin{array}{c}149 \\
(51,7)\end{array}$ & & $\begin{array}{c}254 \\
(88,5)\end{array}$ & $\begin{array}{c}33 \\
(11,5)\end{array}$ & & $\begin{array}{l}263 \\
(92)\end{array}$ & $\begin{array}{l}23 \\
(8)\end{array}$ & \\
\hline $\begin{array}{l}\text { Gravidez de } \\
\text { risco * }\end{array}$ & & & 0,415 & & & 0,510 & & & 0,621 & & & 0,282 \\
\hline Não & $\begin{array}{c}32 \\
(17)\end{array}$ & $\begin{array}{l}156 \\
(83)\end{array}$ & & $\begin{array}{c}90 \\
(47,9)\end{array}$ & $\begin{array}{c}98 \\
(52,1)\end{array}$ & & $\begin{array}{c}161 \\
(86,1)\end{array}$ & $\begin{array}{c}26 \\
(13,9)\end{array}$ & & $\begin{array}{l}190 \\
(96)\end{array}$ & $\begin{array}{c}8 \\
(4)\end{array}$ & \\
\hline Sim & $\begin{array}{c}10 \\
(22,2)\end{array}$ & $\begin{array}{c}35 \\
(77,8)\end{array}$ & & $\begin{array}{c}24 \\
(53,3)\end{array}$ & $\begin{array}{c}21 \\
(46,7)\end{array}$ & & $\begin{array}{c}40 \\
(88,9)\end{array}$ & $\begin{array}{c}5 \\
(11,1)\end{array}$ & & $\begin{array}{c}34 \\
(91,9)\end{array}$ & $\begin{array}{c}3 \\
(8,1)\end{array}$ & \\
\hline
\end{tabular}

* Variável apresentou missing; ** Teste estatístico Qui-Quadrado. 
Tabela 3 - Continuação...

\begin{tabular}{|c|c|c|c|c|c|c|c|c|c|c|c|c|}
\hline \multirow{3}{*}{ Variáveis } & \multicolumn{2}{|c|}{$\begin{array}{c}\text { Aspiração } \\
\text { oronasofaríngea }\end{array}$} & \multirow{3}{*}{$P^{* *}$} & \multicolumn{2}{|c|}{$\begin{array}{l}\text { Aspiração } \\
\text { gástrica }\end{array}$} & \multirow{3}{*}{$P^{* *}$} & \multicolumn{2}{|c|}{$\begin{array}{c}\text { Aspiração } \\
\text { traqueal }\end{array}$} & \multirow{3}{*}{$P^{* *}$} & \multicolumn{2}{|c|}{$\mathrm{O}_{2}$ inalatório } & \multirow{3}{*}{$P^{* *}$} \\
\hline & Não & Sim & & Não & Sim & & Não & Sim & & Não & Sim & \\
\hline & $\begin{array}{c}n \\
(\%)\end{array}$ & $\begin{array}{c}n \\
(\%)\end{array}$ & & $\begin{array}{c}n \\
(\%)\end{array}$ & $\begin{array}{c}n \\
(\%)\end{array}$ & & $\begin{array}{c}n \\
(\%)\end{array}$ & $\begin{array}{c}n \\
(\%)\end{array}$ & & $\begin{array}{c}n \\
(\%)\end{array}$ & $\begin{array}{c}n \\
(\%)\end{array}$ & \\
\hline $\begin{array}{l}\text { Intercorrências } \\
\text { na gestação * }\end{array}$ & & & 0,630 & & & 0,499 & & & 0,684 & & & 0,681 \\
\hline Não & $\begin{array}{c}23 \\
(16,4)\end{array}$ & $\begin{array}{c}117 \\
(83,6)\end{array}$ & & $\begin{array}{c}67 \\
(47,9)\end{array}$ & $\begin{array}{c}73 \\
(52,1)\end{array}$ & & $\begin{array}{c}122 \\
(87,1)\end{array}$ & $\begin{array}{c}18 \\
(12,9)\end{array}$ & & $\begin{array}{c}136 \\
(92,5)\end{array}$ & $\begin{array}{c}11 \\
(7,5)\end{array}$ & \\
\hline Sim & $\begin{array}{c}14 \\
(14,1)\end{array}$ & $\begin{array}{c}85 \\
(85,9)\end{array}$ & & $\begin{array}{c}43 \\
(43,4)\end{array}$ & $\begin{array}{c}56 \\
(56,6)\end{array}$ & & $\begin{array}{c}88 \\
(88,9)\end{array}$ & $\begin{array}{c}11 \\
(11,1)\end{array}$ & & $\begin{array}{c}92 \\
(93,9)\end{array}$ & $\begin{array}{c}6 \\
(6,1)\end{array}$ & \\
\hline Tipo de parto * & & & 0,293 & & & 0,002 & & & 0,867 & & & 0,227 \\
\hline Vaginal & $\begin{array}{c}28 \\
(20,3)\end{array}$ & $\begin{array}{c}110 \\
(79,7)\end{array}$ & & $\begin{array}{c}79 \\
(57,2)\end{array}$ & $\begin{array}{c}59 \\
(42,8)\end{array}$ & & $\begin{array}{c}122 \\
(87,1)\end{array}$ & $\begin{array}{c}17 \\
(12,3)\end{array}$ & & $\begin{array}{c}131 \\
(94,2)\end{array}$ & $\begin{array}{c}8 \\
(5,8)\end{array}$ & \\
\hline Cesárea & $\begin{array}{c}27 \\
(15,7)\end{array}$ & $\begin{array}{c}145 \\
(84,3)\end{array}$ & & $\begin{array}{c}68 \\
(39,5)\end{array}$ & $\begin{array}{c}104 \\
(60,5)\end{array}$ & & $\begin{array}{c}88 \\
(88,9)\end{array}$ & $\begin{array}{c}20 \\
(11,7)\end{array}$ & & $\begin{array}{c}153 \\
(90,5)\end{array}$ & $\begin{array}{c}16 \\
(9,5)\end{array}$ & \\
\hline $\begin{array}{l}\text { Sofrimento } \\
\text { fetal } \\
\text { diagnosticado * }\end{array}$ & & & 0,319 & & & 0,440 & & & 0,619 & & & 0,473 \\
\hline Não & $\begin{array}{c}38 \\
(18,6)\end{array}$ & $\begin{array}{c}166 \\
(81,4)\end{array}$ & & $\begin{array}{c}101 \\
(49,5)\end{array}$ & $\begin{array}{c}103 \\
(50,5)\end{array}$ & & $\begin{array}{c}121 \\
(87,7)\end{array}$ & $\begin{array}{c}25 \\
(12,3)\end{array}$ & & $\begin{array}{c}209 \\
(95,9)\end{array}$ & $\begin{array}{c}9 \\
(4,1)\end{array}$ & \\
\hline Sim & $\begin{array}{c}1 \\
(7,7)\end{array}$ & $\begin{array}{c}12 \\
(92,3)\end{array}$ & & $\begin{array}{c}5 \\
(38,5)\end{array}$ & $\begin{array}{c}8 \\
(61,5)\end{array}$ & & $\begin{array}{c}12 \\
(92,3)\end{array}$ & $\begin{array}{c}1 \\
(7,7)\end{array}$ & & $\begin{array}{c}12 \\
(100)\end{array}$ & $\begin{array}{c}0 \\
(0)\end{array}$ & \\
\hline $\begin{array}{l}\text { Alteração no } \\
\text { exame físico * }\end{array}$ & & & 0,049 & & & 0,584 & & & 0,461 & & & 0,000 \\
\hline Não & $\begin{array}{c}42 \\
(20,4)\end{array}$ & $\begin{array}{c}164 \\
(79,6)\end{array}$ & & $\begin{array}{c}94 \\
(45,6)\end{array}$ & $\begin{array}{c}112 \\
(54,4)\end{array}$ & & $\begin{array}{c}178 \\
(86,8)\end{array}$ & $\begin{array}{c}27 \\
(13,2)\end{array}$ & & $\begin{array}{c}192 \\
(96,5)\end{array}$ & $\begin{array}{c}7 \\
(3,5)\end{array}$ & \\
\hline Sim & $\begin{array}{c}11 \\
(11,2)\end{array}$ & $\begin{array}{c}87 \\
(88,8)\end{array}$ & & $\begin{array}{c}48 \\
(49)\end{array}$ & $\begin{array}{l}50 \\
(51)\end{array}$ & & $\begin{array}{c}88 \\
(89,8)\end{array}$ & $\begin{array}{c}10 \\
(10,2)\end{array}$ & & $\begin{array}{c}84 \\
(83,2)\end{array}$ & $\begin{array}{c}17 \\
(16,8)\end{array}$ & \\
\hline $\begin{array}{l}\text { Manobras de } \\
\text { reanimação * }\end{array}$ & & & 0,009 & & & 0,265 & & & 0,031 & & & 0,000 \\
\hline Não & $\begin{array}{c}47 \\
(18,7)\end{array}$ & $\begin{array}{c}204 \\
(81,3)\end{array}$ & & $\begin{array}{c}119 \\
(47,4)\end{array}$ & $\begin{array}{c}132 \\
(52,6)\end{array}$ & & $\begin{array}{c}216 \\
(86,4)\end{array}$ & $\begin{array}{c}34 \\
(13,6)\end{array}$ & & $\begin{array}{c}278 \\
(100)\end{array}$ & $\begin{array}{c}0 \\
(0)\end{array}$ & \\
\hline Sim & $\begin{array}{c}0 \\
(0)\end{array}$ & $\begin{array}{c}30 \\
(100)\end{array}$ & & $\begin{array}{c}11 \\
(36,7)\end{array}$ & $\begin{array}{c}19 \\
(63,3)\end{array}$ & & $\begin{array}{c}30 \\
(100)\end{array}$ & $\begin{array}{c}0 \\
(0)\end{array}$ & & $\begin{array}{c}9 \\
(27,3)\end{array}$ & $\begin{array}{c}24 \\
(72,7)\end{array}$ & \\
\hline
\end{tabular}

* Variável apresentou missing; ** Teste estatístico Qui-Quadrado.

alterações, assim como os recém-nascidos de parto cesáreo $(p=0,045 ; O R=0,55 ; I C: 0,313-0,986)$, em comparação aos de parto vaginal. No que tange às práticas invasivas, apenas o tipo de parto manteve-se associado à aspiração gástrica, indicando que nascer de parto cesáreo aumentou em duas vezes as chances de o recém-nascido ser submetido a essa intervenção invasiva $(p=0,002 ; O R=2,04 ; I C: 1,299-3,228)$, em relação aos nascidos de parto vaginal (Tabela 4 ).
Dentre as práticas assistenciais, o contato com o seio materno com alojamento conjunto ( $p=0,032)$ e aspiração traqueal $(p=0,015)$, e alojamento conjunto com oxigênio inalatório $(p=0,000)$ e aspiração oronasofaríngea $(p=0,015)$ apresentaram associação. A chance de ir para o Alojamento Conjunto acompanhado de sua mãe logo após a saída da sala de parto foi aproximadamente 4,5 vezes maior entre recém-nascidos colocados em contato com o seio materno ( $p=0,048 ; O R=4,46 ; I C: 1,016-19,658)$, em relação aos que não 
Tabela 4 - Odds Ratio pela regressão logística binária simples para as práticas assistenciais na sala de parto $(\mathrm{N}=351)$. Rio das Ostras, RJ, Brasil, 2019.

\begin{tabular}{|c|c|c|}
\hline \multicolumn{3}{|c|}{ Contato com o seio materno } \\
\hline Variáveis & OR (IC95\%) & Valor de $p$ \\
\hline $\begin{array}{l}\text { Alterações no } \\
\text { exame físico }\end{array}$ & $0,32(0,167-0,632)$ & 0,001 \\
\hline Parto Cesáreo & $0,55(0,313-0,986)$ & 0,045 \\
\hline Aspiração Traqueal & $0,11(0,15-0,901)$ & 0,039 \\
\hline \multicolumn{3}{|c|}{ Aspiração Gástrica } \\
\hline Variável & OR (IC95\%) & Valor de $p$ \\
\hline Parto Cesáreo & $2,04(1,299-3,228)$ & 0,002 \\
\hline \multicolumn{3}{|c|}{ Alojamento Conjunto } \\
\hline Variável & OR (IC95\%) & Valor de $p$ \\
\hline $\begin{array}{c}\text { Contato com o seio } \\
\text { materno }\end{array}$ & $4,46(1,016-19,658)$ & 0,048 \\
\hline
\end{tabular}

ficaram nessa posição. Por outro lado, a aspiração traqueal diminuiu as chances desse contato $(p=0,039 ; O R=0,11 ; I C: 0,15-0,901)$, quando comparada aos que não foram submetidos a essa intervenção invasiva (Tabela 4).

\section{DISCUSSÃo}

O presente estudo possibilitou identificar fatores associados às práticas assistenciais ao recém-nascido na sala de parto. Houve predomínio de mães adultas jovens, contudo, aproximadamente um terço das mulheres pertencia às faixas etárias consideradas de risco gestacional, pois eram adolescentes ou por possuírem 35 anos ou mais. Em relação à escolaridade, uma parcela significativa não havia completado o ciclo regular do ensino obrigatório. Essas variáveis sociodemográficas maternas são apontadas como fatores correlacionados à assistência ao recém-nascido a termo na sala de parto. Os extremos de idade da vida reprodutiva, como a adolescência, diminuem as chances de contato pele a pele precoce e de amamentação na primeira hora de vida. ${ }^{2}$ Porém, apesar do baixo percentual dessas práticas no cenário investigado, não houve associações entre essas variáveis no presente estudo.

Sobre as condições de gestação, a grande maioria das mães vivenciou gestações únicas, com acompanhamento no pré-natal de baixo risco e mais da metade não apresentou nenhum tipo de intercorrência ao longo da gestação. Dentre as que realizaram pré-natal, em torno de dois terços compareceram a seis ou mais consultas. A gestação única ${ }^{14} \mathrm{e}$ assistência pré-natal, com número adequado de consultas, ${ }^{15,16}$ são fatores relevantes para a amamentação na primeira hora de vida, entretanto não houve significância estatística entre os resultados atuais.

Quanto ao tipo de parto, a cesariana foi mais recorrente entre os nascimentos, bem acima dos $15 \%$ recomendados pela OMS, assim como em outros estudos brasileiros. ${ }^{17-19}$ Altas taxas de cesariana, como a encontrada, estão relacionadas, dentre outros aspectos, aos recursos tecnológicos e humanos disponíveis, e, especialmente, aos protocolos clínicos adotados nas unidades de saúde. Porém, incluem o desejo da mulher por essa via de parto no início da gravidez, a realização de cesárea prévia, bem como as orientações prévias. ${ }^{18-20}$

O tipo de parto interfere nas práticas adotadas na atenção hospitalar ao recém-nascido saudável. ${ }^{2}$ De igual forma, o contato com o seio materno precoce esteve associado ao tipo de parto, bem como demonstraram outros estudos, ${ }^{2,7,21}$ reforçando que o parto vaginal tem efeito protetor contra o atraso no início da amamentação, quando comparado à cesárea, que, consequentemente, está relacionada ao desmame precoce e ao menor tempo de aleitamento materno exclusivo. ${ }^{22}$

Assim, o parto cesáreo se apresentou como um fator de risco para a não realização desse contato, bem como a existência de alterações no exame físico, pois ambos podem reduzir as chances dessa prática. ${ }^{2,7,21}$ Tem-se, ainda, que os achados não evidenciaram associação entre o tipo de parto e o uso de oxigênio inalatório, como em outro estudo, ${ }^{2}$ mas à aspiração gástrica. Constatou-se que o parto cesáreo é um fator fortemente associado a essa intervenção invasiva.

Dentre os recém-nascidos, predominaram os do sexo feminino de raça/cor branca, o que reflete a distribuição percentual por sexo do último Censo Demográfico Brasileiro em 2010, que apresentou predominância de $51,0 \%$ do sexo feminino. Pequena parcela estava registrada como preta, revelando uma característica atípica nessa maternidade pública localizada na região Sudeste, pois o estado do Rio de Janeiro se destaca em relação aos outros, por contar com $12,4 \%$ de pessoas de raça ou cor preta, com aproximadamente dois milhões de pessoas. ${ }^{23}$

$\mathrm{Na}$ declaração de nascidos vivos, é utilizada a hétero classificação, isto é, outra pessoa, geralmente um familiar, define a cor/raça do bebê, ${ }^{24}$ sendo este um dos critérios utilizados em pesquisas para medir diferenças sociais, tratamentos e desfechos em saúde, inclusive obstétricos e neonatais. ${ }^{25}$ Estudo brasileiro de base populacional concluiu que, em comparação às brancas, puérperas pretas ou pardas possuíram maior risco de terem um pré-natal inadequado e ausência de acompanhante, reforçando as iniquidades raciais, inclusive nas práticas assistenciais na sala de parto, ${ }^{26}$ o que avigora a importância quanto ao preenchimento adequado dessa informação.

A grande maioria era de recém-nascidos a termo, sem baixo peso ao nascer e com peso adequado para idade gestacional. Uma parcela expressiva apresentou ausência de dificuldade para adaptação à vida extrauterina segundo o Apgar no $1^{\circ}$ e no $5^{\circ}$ minuto, com raros casos de sofrimento fetal. Logo, o perfil encontrado foi de recém-nascidos saudáveis que deveriam ser assistidos junto de suas mães, sem a necessidade de intervenções invasivas, como as identificadas (aspiração oronasofaríngea, gástrica e traqueal e oxigênio inalatório). Pelas Diretrizes da Sociedade Brasileira de Pediatria, baseadas nas melhores evidências disponíveis, o recém-nascido que apresenta boa vitalidade deve permanecer junto de sua mãe depois do clampeamento 
do cordão umbilical. Ainda, na sala de parto, enquanto estiver junto à sua mãe, é preciso apenas prover calor, manter as vias aéreas pérvias e avaliar a sua vitalidade de maneira continuada. ${ }^{5}$

Dentre as práticas na sala de parto, comprovadamente benéficas ao recém-nascido, verificou-se baixo percentual de contato pele a pele e com o seio materno precoce, o que foi parcialmente compatível com um estudo nacional de base hospitalar, no qual o contato pele a pele logo após o nascimento foi de $28,2 \%$, mas a oferta do seio materno na sala de parto de apenas $16,1 \%$, revelando índice menor que o estudo atual. ${ }^{2}$ Pesquisa realizada no Nordeste apresentou valores ainda mais baixos, ao apontar que apenas 9,3\% das mães permaneceram em contato com seu bebê por mais de 30 minutos ou até a primeira mamada. ${ }^{21}$ Outra investigação realizada em São Paulo, em uma instituição intitulada Amiga da Criança, apresentou 35,0\% dos recém-nascidos colocados em contato pele a pele imediato, porém apenas $9,3 \%$ foram amamentados precocemente, ${ }^{27}$ o que reforça que as variações entre as regiões e estados brasileiros persistem.

A prática de secagem, recomendada para a manutenção da normotermia, ${ }^{5}$ apresentou melhor taxa, quando comparada com investigação realizada na Índia, que obteve $82,0 \%{ }^{28} \mathrm{~A}$ prática de Alojamento Conjunto direto da sala de parto também foi superior ao encontrado no estudo brasileiro, tanto no Brasil como um todo, que foi de $69,0 \%$, sendo maior do que todas as regiões brasileiras separadamente, a exemplo da própria região Sudeste, que foi de $61,4 \%{ }^{2}$

Mesmo diante de recém-nascidos saudáveis, as taxas de intervenções invasivas, identificadas na sala parto, são elevadas, especialmente as aspirações oronasofaríngea e gástrica, que apresentaram maiores frequências, com valores superiores à média nacional, de $71,0 \%$ e $39,5 \%$, respectivamente, ${ }^{2}$ bem como de outra investigação conduzida no centro obstétrico de um hospital universitário público do Sul do Brasil, cujas taxas foram $54,9 \%$ e $18,9 \%{ }^{29}$

Pesquisas corroboram que práticas sem comprovação científica de benefícios efetivos aos recém-nascidos ainda são comuns, ${ }^{2,28,29}$ o que indica que as novas diretrizes para atendimento ao neonato saudável ainda não foram totalmente incorporadas à prática clínica. ${ }^{2}$ Porém, estudo que analisou a assistência antes e após uma intervenção com profissionais de saúde que assistem ao parto e nascimento apresentou taxas de aspiração de vias aéreas superiores após o parto de $75,0 \%$, mas, após a intervenção, essa proporção diminuiu consideravelmente para $34,0 \%,{ }^{28}$ reforçando a importância de estratégias junto aos profissionais para reversão desse cenário.

A única variável que permaneceu como associada à aspiração gástrica foi o tipo de parto, na medida em que nascer de cesariana aumentou expressivamente as chances de o recém-nascido ser submetido a esse tipo de aspiração, duas práticas que precisam ser reduzidas. Revisão integrativa de estudos com bebês sadios a termo indicou que não houve diferenças significativas nos resultados de saúde entre bebês submetidos à aspiração orofaríngea e os não aspirados, o que também foi observado entre bebês que utilizaram oxigênio $e$ aqueles mantidos em ar ambiente, portanto, 0 abandono dessas práticas é apoiado. A aspiração gástrica é prejudicial e não causa benefícios aos recém-nascidos saudáveis, logo, não deveria ser usada. Na maioria dos casos, quanto menos intervenções, melhor para o bebê, ou seja, as recomendações apoiam um nascimento suave e fisiológico, com atendimento centrado no recém-nascido e na família. ${ }^{30}$

Chama atenção o fato de que a realização de contato pele a pele precoce $\mathrm{e} o$ aleitamento materno ainda na sala de parto estiveram associados à maior probabilidade da ida do bebê para o alojamento conjunto. Esses dados corroboram estudo realizado em Minas Gerais, o qual demonstrou uma associação positiva entre as boas práticas ao nascimento, contato pele a pele e aleitamento materno precoce e o encaminhamento imediato ao alojamento conjunto. ${ }^{2}$

Logo, nascer de parto normal, ter boa vitalidade, sofrer menos intervenções invasivas, especialmente a aspiração gástrica, contribui para o contato pele a pele e a oferta do seio na sala de parto, o que aumenta as chances da ida imediata ao Alojamento Conjunto, reforçando a importância de práticas assistenciais humanizadas e menos intervencionistas que garantam o respeito à fisiologia do nascimento. Portanto, é preciso galgar avanços na aplicabilidade de práticas notadamente benéficas na área obstétrica e neonatal, objetivando reduzir intervenções desnecessárias, garantindo, assim, uma experiência positiva para o binômio. ${ }^{31}$

\section{CONCLUSÃO E IMPLICAÇÕES PARA A PRÁTICA}

O tipo de parto e as alterações no exame físico foram os principais fatores associados às práticas assistenciais ao recém-nascido na sala de parto. $O$ parto cesáreo diminui as chances de contato com o seio materno e aumenta as chances de aspiração gástrica. Apresentar alterações no exame físico reduz as chances de contato com o seio materno. Os achados reafirmam o modelo de atenção ao parto e nascimento do Brasil, no qual prevalece o parto cesáreo e as intervenções invasivas, sem o devido embasamento nas melhores evidências científicas disponíveis.

Este estudo tem limitação quanto às suas informações, dado o único contexto geográfico definido, sendo necessária a realização de mais estudos locais que apresentem realidades diversas, além de pesquisas voltadas à identificação dos motivos para a ocorrência ou não das práticas comprovadamente benéficas ao recém-nascido. Ademais, o caráter transversal do estudo impossibilita o estabelecimento de uma relação causal para as práticas assistenciais realizadas.

Os resultados apresentaram os fatores relacionados às práticas assistenciais, recomendadas e não recomendadas, realizadas na sala de parto, focadas integralmente no recém-nascido, sendo esse o principal diferencial da pesquisa. A identificação desses fatores possibilita a organização das rotinas dos serviços, 
de modo a evitar intervenções desnecessárias e visando uma atenção obstétrica e neonatal humanizada e de qualidade, com práticas assistenciais baseadas em evidências, conforme critérios estabelecidos pelo Ministério da Saúde e a OMS.

\section{FINANCIAMENTO}

O presente estudo foi financiado através da Bolsa de Iniciação Científica pela Fundação de Amparo à Pesquisa do Estado do Rio de Janeiro (FAPERJ), concedida a Mayara Pacheco da Conceição Bastos, Processo E-26/200.562/2019.

\section{CONTRIBUIÇÕES DOS AUTORES}

Desenho do estudo, aquisição, análise de dados e interpretação dos resultados, redação e revisão crítica do manuscrito, aprovação da versão final do artigo, responsabilidade por todos os aspectos do conteúdo e a integridade do artigo publicado: Beatriz Cabral Ledo, Andressa Silva Torres dos Santos e Mayara Pacheco da Conceição Bastos.

Desenho do estudo, interpretação dos resultados e revisão crítica do manuscrito, aprovação da versão final do artigo, responsabilidade por todos os aspectos do conteúdo e a integridade do artigo publicado: Fernanda Garcia Bezerra Góes, Fernanda Maria Vieira Pereira-Ávila e Aline Cerqueira Santos Santana da Silva.

\section{EDITOR ASSOCIADO}

\section{Aline Cristinane Cavicchioli Okido}

\section{REFERÊNCIAS}

1. Chaves RL. O nascimento como experiência radical de mudança. Cad Saude Publica. 2014;30(Supl 1):S14-6. http://dx.doi.org/10.1590/0102311XPE03S114. PMid:25167173.

2. Moreira MEL, Gama SGN, Pereira APE, Silva AAM, Lansky S, Pinheiro RS et al. Práticas de atenção hospitalar ao recém-nascido saudáve no Brasil. Cad Saude Publica. 2014;30(Supl 1):S128-39. http://dx.doi. org/10.1590/0102-311X00145213. PMid:25167172.

3. Ramos WMA, Aguiar BGC, Conrad D, Pinto CB, Mussumeci PA Contribuição da enfermeira obstétrica nas boas práticas da assistência ao parto e nascimento. Rev. Pesqui. (Univ. Fed. Estado Rio J., Online) [Internet]. 2018 jan/mar; [citado 2020 jan 25];10(1):173-9. Disponível em: https://pesquisa.bvsalud.org/portal/resource/pt/bde-32254

4. Andrade LO, Felix ESP, Souza FS, Gomes LOS, Boery RNSO. Práticas dos profissionais de enfermagem diante do parto humanizado. Rev Enferm UFPE Online [Internet]. 2017; [citado 2020 jan 25];11(Supl 6):2576-85. Disponível em: https://pesquisa.bvsalud.org/portal/resource/ pt/biblio-1032487

5. Sociedade Brasileira de Pediatria. Reanimação do recém-nascido $\geq 34$ semanas em sala de parto: Diretrizes 2016 da Sociedade Brasileira de Pediatria [Internet]. 2016 [citado 2020 jan 25]. Disponível em: https:// www.sbp.com.br/especiais/reanimacao-neonatal/

6. Moore ER, Bergman N, Anderson GC, Medley N. Early skin-to-skin contact for mothers and their healthy newborn infants. Cochrane Database Syst Rev. 2016;11(CD003519):CD003519. http://dx.doi. org/10.1002/14651858.CD003519.pub4. PMid:27885658.

7. Kologeski TK, Strapasson MR, Schneider V, Renosto JM. Contato pele a pele do recém-nascido com sua mãe na perspectiva da equipe multiprofissional. Rev Enferm UFPE Online. 2017;11(1):94-101. http:// dx.doi.org/10.5205/reuol.9978-88449-6-1101201712.
8. Cetinkaya E, Ertem G. The effects of skin-to-skin contact on maternalpreterm infants: a systematic review. J Educ Res Nurs. 2017;14(2):16775. http://dx.doi.org/10.5222/HEAD.2017.167.

9. Rocha LB, Araujo FMS, Rocha NCO, Almeida CD, Santos MO, Rocha $\mathrm{CHR}$. Aleitamento materno na primeira hora de vida: uma revisão da literatura. Rev Med Saúde Brasília [Internet]. 2017; [citado 2020 jan 25];6(3):384-94. Disponível em: https://portalrevistas.ucb.br/index.php/ rmsbr/article/view/8318/5490

10. Khan J, Vesel L, Bahl R, Martines JC. Timing of breastfeeding initiation and exclusivity of breastfeeding during the first month of life: effects on neonatal mortality and morbidity - a systematic review and meta-analysis. Matern Child Health J. 2015;19(3):468-79. http://dx.doi.org/10.1007/ s10995-014-1526-8. PMid:24894730.

11. Monguilhott JJDC, Brüggemann OM, Freitas PF, d'Orsi E. Nascer no Brasil: a presença do acompanhante favorece a aplicação das boas práticas na atenção ao parto na região Sul. Rev Saude Publica. 2018;52:1. http://dx.doi.org/10.11606/S1518-8787.2018052006258. PMid:29364356.

12. Santos LM, Silva JCR, Carvalho ESS, Carneiro AJS, Santana RCB Fonseca MCC. Vivenciando o contato pele a pele com o recém-nascido no pós-parto como um ato mecânico. Rev Bras Enferm. 2014;67(2):2027. http://dx.doi.org/10.5935/0034-7167.20140026.

13. Pereira SB, Diaz CMG, Backes MTS, Ferreira CLL, Backes DS. Boas práticas de atenção ao parto e ao nascimento na perspectiva de profissionais de saúde. Rev Bras Enferm. 2018;71(Supl 3):1313-9. http://dx.doi.org/10.1590/0034-7167-2016-0661. PMid:29972529.

14. Kim B-Y. Factors that influence early breastfeeding of singletons and twins in Korea: a retrospective study. Int Breastfeed J. 2017;12(4):1-10. http:// dx.doi.org/10.11606/S1518-8787.2018052006258. PMid:28074106.

15. Ndirangu MN, Gatimu SM, Mwinyi HM, Kibiwott DC. Trends and factors associated with early initiation of breastfeeding in Namibia: analysis of the demographic and health surveys 2000-2013. BMC Pregnancy Childbirth. 2018;18(1):171. http://dx.doi.org/10.1186/s12884-018-18114. PMid:29769063.

16. Berde AS, Yalcin SS. Determinants of early initiation of breastfeeding in Nigeria: a population-based study using the 2013 demograhic and health survey data. BMC Pregnancy Childbirth. 2016;16(1):32. http:// dx.doi.org/10.1186/s12884-016-0818-y. PMid:26852324.

17. Santos JB, Souza EM, Rocha CS, Trindade FS, Oliveira KA. Aspectos epidemiológicos do parto cesáreo em Sergipe. Rev Saúde ReAGES [Internet]. 2019; [citado 2020 jan 25];1(4):47-51. Disponível em: http:// npu.faculdadeages.com.br/index.php/revistadesaude/article/view/168

18. Vale LD, Lucena EEES, Holanda CSM, Cavalcante RD, Santos MM Preferência e fatores associados ao tipo de parto entre puérperas de uma maternidade pública. Rev Gaúcha Enferm. 2015 jul/set;36(3):86-92. http://dx.doi.org/10.1590/1983-1447.2015.03.50032. PMid:26486904

19. Oliveira RR, Melo EC, Novaes ES, Ferracioli PLRV, Mathias TAF. Factors associated to cesarean delivery in public and private health care systems. Rev Esc Enferm USP. 2016;50(5):733-40. http://dx.doi. org/10.1590/s0080-623420160000600004. PMid:27982390.

20. Silva ACL, Félix HCR, Ferreira MBG, Wysocki AD, Contim D, Ruiz MT. Preferência pelo tipo de parto, fatores associados à expectativa e satisfação com o parto. Rev Eletr Enf. 2017;19:a34. http://dx.doi. org/10.5216/ree.v19.44139.

21. Sampaio ARR, Bousquat A, Barros C. Contato pele a pele ao nascer: um desafio para a promoção do aleitamento materno em maternidade pública no nordeste brasileiro com o título de Hospital Amigo da Criança. Epidemiol Serv Saude. 2016;25(2):281-90. https://doi.org/10.5123/ s1679-49742016000200007. PMid:27869946.

22. Arruda GT, Barreto SC, Morin VL, Petter GN, Braz MM, Pivetta HMF Existe relação da via de parto com amamentação na primeira hora de vida? Rev Bras Promoc Saúde. 2018;31(2):1-7. http://dx.doi. org/10.5020/18061230.2018.7321.

23. Instituto Brasileiro de Geografia e Estatística. Censo demográfico 2010: características da população e dos domicílios. Resultados do universo [Internet]. 2011 [citado 2020 jan 25]. Disponível em: https://censo2010. ibge.gov.br/resultados.html

24. Ministério da Saúde (BR). Guia de implementação do quesito Raça/ Cor/Etnia [Internet]. Brasília: Ministério da Saúde; 2018 [citado 2020 
jan 25]. Disponível em: http://bvsms.saude.gov.br/bvs/publicacoes/ guia_implementacao_raca_cor_etnia.pdf

25. Pacheco VC, Silva JC, Mariussi AP, Lima MR, Silva TR. As influências da raça/cor nos desfechos obstétricos e neonatais desfavoráveis. Saúde Debate. 2018;42(116):125-37. http://dx.doi.org/10.1590/0103-1104201811610.

26. Leal MC, Gama SGN, Pereira APE, Pacheco VE, Carmo CN, Santos RV. A cor da dor: iniquidades raciais na atenção pré-natal e ao parto no Brasil. Cad Saude Publica. 2017;33(33, Supl 1):e00078816. http:// dx.doi.org/10.1590/0102-311x00078816. PMid:28746555.

27. Calegari FL, Barbieratto BJ, Fujinaga Cl, Fonseca LMM, Oliveira CR, Leite AM. Prontidão do recém-nascido a termo durante a primeira mamada em alojamento conjunto. Rev Rene. 2016 out 4;17(4):444-50. http://dx.doi.org/10.15253/2175-6783.2016000400002.

28. lyengar K, Jain M, Thomas S, Dashora K, Liu W, Saini P et al. Adherence to evidence based care practices for child birth before and after a quality improvement intervention in health facilities of Rajasthan, India. BMC
Pregnancy Childbirth. 2014;14(1):270. http://dx.doi.org/10.1186/14712393-14-270. PMid:25117856.

29. Abdala LG, Cunha MLC. Contato pele a pele entre mãe e recém-nascido e amamentação na primeira hora de vida. Clin Biomed Res. 2018;38(4):356-60. http://dx.doi.org/10.4322/23579730.82178

30. Mercer JS, Erickson-Owens DA, Graves B, Haley MM. Práticas baseadas em evidências para a transição do feto a recém-nascido. Rev Tempus Actas Saúde Col. [Internet]. 2010; [citado 2020 jan 25];4(4):173-89. Disponível em: http://redehumanizasus.net/acervo/praticas-baseadasem-evide\%CC\%82ncias-para-a-transic\%CC\%A7a\%CC\%83o-de-fetoa-recem-nascido/

31. Sandall J, Soltani H, Gates S, Shennan A, Devane D. Midwife-led continuity models versus other models of care for childbearing women. Cochrane Database Syst Rev. 2016;4(CD004667):CD004667. http:// dx.doi.org/10.1002/14651858.CD004667.pub5. PMid:27121907. 